\title{
REGIÓN Y TERRITORIO. Aspectos conceptuales en torno a la organización del espacio y el rol de la ciencia geográfica.
}

\section{REGION AND TERRITORY. Conceptual aspects about space organization and the role of geographical science.}

\section{Resumen}

Cuadra, Dante Edin dantecuadra@yahoo.com

Lucca, Amalia ailucca@hum.nne.edu.ar Taborda, Marta Beatriz tabordamarta@yahoo.com.ar

Combaz, Silvia Fernanda f combaz@hotmail.com

Romero, Luis María luis ro mero@hotmail.com Instituto de Geografía de la UNNE

La Geografía es la ciencia que tiene como dominio a la superficie terrestre y como objeto de estudio al espacio geográfico. Según las épocas transcurridas, el énfasis ha sido puesto sobre el paisaje, las relaciones sociedad-naturaleza, la región, el territorio o el lugar. Cualquiera fuese el enfoque, a la Geografía -como ciencia humanística y social- le interesa estudiar el espacio terrestre no per se, sino proyectando condiciones, configuraciones y ordenaciones tendientes a la mejor calidad de vida de quienes habitan una porción territorial en la escala que se considere (local, comarcal, provincial, regional e, incluso, global). Por lo tanto, la planificación y el ordenamiento territorial, si bien han sido un tanto desatendidos por los geógrafos durante largas etapas y en muchos países, en la actualidad vuelve a cobrar una gran significación, hasta tornarse un desafío crucial y una responsabilidad ineludible, que no pueden dejarse en manos -solamente- de otras disciplinas.

Nuestro mundo, cada vez más habitado, explotado, complejo y conflictivo, reclama nuevas miradas, nuevos órdenes, organizaciones más justas e inclusivas. No es posible que la ciencia de la espacialidad se abstenga ante tamaño reto: la Geografía tiene historia, contenidos, métodos, técnicas y recursos humanos para aportar, junto con otras ciencias, muchas de las soluciones que las sociedades del siglo XXI requieren con desesperación.

La reflexión en torno a algunos conceptos elementales de la organización del espacio, quizás nos lleve a reenfocar nuestro quehacer cotidiano y a cultivar nuestra ciencia de un modo más impactante en términos sociales y ambientales.

\section{Palabras claves}

Organización del espacio. Región geográfica. Planificación. Ordenamiento territorial.

\begin{abstract}
Geography is the science which domain is the Earth surface and that aims to study geographical space. According to the elapsed times, the emphasis has been placed on the concepts of landscape, relationship between society and nature, region, territory or place. Whatever the approach, Geography
\end{abstract}

Publicado en formato digital: Dr. Dante Edín Cuadra y otros. REGIÓN Y TERRITORIO. Aspectos conceptuales en torno a la organización del espacio y el rol de la ciencia geográfica. Revista Geográfica Digital. IGUNNE. Facultad de Humanidades. UNNE. Año 12. № 24. Julio - Diciembre 2015. ISSN 1668-5180 Resistencia, Chaco. 
-as a social and humanistic science- is interested in the study of terrestrial space not per se, but projecting conditions, configurations and arrangements aimed at improving the quality of life of those who inhabit a land portion on the scale considered (local, regional, and even global). Therefore, territorial planning, which has been somewhat neglected by geographers during long stages and in many countries, now returns to collect a great significance, to become a crucial challenge and an inescapable responsibility that can not be left in the hands of other disciplines -only-.

Our world, which is increasingly populated, exploited, complex and conflicting, demands new perspectives, new orders and more just and inclusive organizations.

It is not possible that the science of space refrains to take up such a big challenge: Geography has a history, contents, methods, techniques and human resources to contribute, along with other sciences, to many of the solutions that the XXI century desperately requires.

The reflection on some basic concepts of the organization of space, perhaps take us to refocus our daily lives and our science to grow more impacting in social and environmental terms.

\section{Keywords}

Organization of space. Geographic region. Territorial planning. Land use.

\section{Objetivos}

- Organizar una reseña conceptual sobre región y territorio a partir de los aportes de diferentes autores de distintas épocas.

- Examinar los significados y alcances de diversos conceptos vinculados a la organización regional y territorial.

- Reflexionar sobre el rol de la Geografía en las problemáticas regionales y territoriales. 


\section{Introducción}

Este trabajo se inserta en el contexto del Proyecto Institucional que lleva adelante el Instituto de Geografía para el período 2014-2017, que fuera acreditado por la Secretaría General de Ciencia y Técnica de la Universidad Nacional del Nordeste (UNNE) con el código H001-2013. En esta contribución se pretende echar luz y reposicionar algunos conceptos considerados fundamentales para el desarrollo regional y el ordenamiento territorial.

Ordenar sería identificar, distribuir, organizar y regular las actividades humanas en un territorio, con la aspiración de alcanzar el equilibrio entre eficacia y equidad de una sociedad, a través de la proyección espacial de diferentes políticas que posibilitan formular planes (en diferentes niveles) y gestionar (mediante una administración) con el fin de mejorar la calidad de vida de la población. En su aplicación se contemplan tres elementos: actividades humanas, espacio en el que se ubican y sistema que ambos conforman.

El grupo de trabajo se ha propuesto brindar un soporte teórico clarificador sobre la implicancia de algunos conceptos y volver a poner en el centro de la escena a la temática de la "organización del espacio" que, a veces, ante tantas variantes desarrolladas en el interior del campo geográfico y fuera de él, parecen diluir la esencia misma del objeto de estudio de nuestra disciplina. También, nos hemos decidido a exponer algunas reflexiones en torno al rol que le compete a la ciencia geográfica en esta temática -atravesada por una diversidad de disciplinas- al promediar la segunda década del siglo XXI.

Como se sabe, la organización del espacio ha sido, y sigue siendo, una preocupación esencial entre los geógrafos, por tratarse de una temática inherente al propio objeto de estudio de la disciplina.

Justamente, la Geografía no estudia los elementos, distribuciones, relaciones, factores y procesos que ocurren en la superficie terrestre por mera curiosidad o con la sola intención de ampliar el repositorio de conocimientos a través de libros, revistas, atlas y otras formas de divulgación. La esencia de la disciplina está orientada a entender e interpretar cómo se configura el espacio geográfico en distintas escalas (local, comarcal, regional, nacional o mundial), para luego evaluar qué aspectos podrían modificarse o reordenarse en vistas a que sus habitantes alcancen una mejor calidad de vida en un escenario que garantice la equidad entre los grupos humanos que lo habitan y no se afecten los equilibrios ecosistémicos del planeta. Benko (1999) reconoció que han sido los geógrafos quienes desde fines del siglo XIX “...estudiaron la organización territorial de las sociedades."

Actualmente, existe una proliferación de textos en los que aparecen términos que generan una gran confusión entre los lectores, tratándose muchas veces de conjugaciones distintas con significados semejantes. Es así como aparecen conceptos vinculados con el territorio, como ordenación, ordenamiento, planificación, planeamiento, planeación, plan estratégico, gestión, manejo, organización y desarrollo regional, entre otros.

En realidad, la connotación de estos conceptos no es nueva, ya que desde la Geografía Sistemática, la Geografía Regional en particular y, desde otras disciplinas, históricamente se transfirieron los saberes, en la medida de sus posibilidades, a los organismos públicos de los distintos países encargados de la gestión, planificación, ordenación y desarrollo de sus territorios a distintas escalas: nacional, regional, provincial, ejidal o urbana. Lo nuevo, ha sido el gran cambio de paradigma acaecido desde los años '80 en adelante, cuando se desarrollaron y difundieron nuevas técnicas y tecnologías, herramientas digitales y remotas que han posibilitado disponer y procesar una enorme cantidad de datos en poco tiempo y, esos mismos dispositivos, han permitido posteriormente una propagación y construcción comunitaria de la información a nivel global (desarrollo teórico), como nunca antes había ocurrido.

De hecho, las diferentes sociedades a lo largo de la historia han planificado sus ciudades, los usos que iban a dar a sus tierras, la localización de sus instituciones y de los espacios públicos, la implementación de infraestructura, etc. El manejo hidráulico realizado por los Incas, las obras viales y acueductos de los romanos y la organización de ciudades como Babilonia, Granada o Córdoba (estas

Publicado en formato digital: Dr. Dante Edín Cuadra y otros. REGIÓN Y TERRITORIO. Aspectos conceptuales en torno a la organización del espacio y el rol de la ciencia geográfica. Revista Geográfica Digital. IGUNNE. Facultad de Humanidades. UNNE. Año 12. N N 24. Julio - Diciembre 2015. ISSN 1668-5180 Resistencia, Chaco. 
dos últimas en España), son sólo algunos ejemplos de planificación e intervención territorial en el contexto técnico y cultural de cada época.

Nuestro tiempo, en el tránsito de la segunda década del siglo XXI, se caracteriza por las disparidades, asimetrías y desequilibrios en todo orden y escala: así como muchos países, en especial de Latinoamérica, han podido recuperar su vida democrática en las últimas décadas, otras áreas del mundo sufren los excesos e injusticias ejercidos por regímenes dictatoriales, grupos fundamentalistas, guerras e intolerancia religiosa, política, racial y cultural, como observamos en Siria, Irak, Yemen, Libia, Somalia, Eritrea y Nigeria, entre otros. También asistimos al "...incremento de las desigualdades regionales, sociales y territoriales asociadas al avance de la globalización." (Precedo Ledo, 2004) A ello se suma el incremento de la población, sobre todo en los países subdesarrollados, el abarrotamiento de las ciudades, las migraciones masivas impulsadas por superpoblación, guerras, persecuciones políticas y religiosas, los desastres naturales y el agotamiento de sistemas productivos. En consecuencia, vivimos una época en la que muchos territorios se desordenan, se fracturan y colapsan, se profundizan las desigualdades regionales y, además, los ordenamientos logrados anteriormente bajo otros paradigmas entran en desequilibrio. "...el espacio y el tiempo se han comprimido, las distancias se han relativizado y las barreras espaciales se han suavizado. Sin embargo, aunque ello sea así, es realmente paradójico que el espacio -o más específicamente el territorio- no sólo no haya perdido importancia, sino que ha aumentado su influencia y su peso específico." (Nogué y Rufí, 2001. En: Precedo Ledo, 2004)

A continuación se presenta una revisión de algunos conceptos que confluyen en la temática expuesta, como espacio geográfico, región, planificación, territorios y ordenamiento territorial, que cuentan con un amplio tratamiento teórico por parte de diferentes autores de la ciencia geográfica y de otras disciplinas.

\section{Desarrollo}

\section{Espacio geográfico, el punto de partida. Región geográfica, un concepto difuso}

Von Richthofen postuló en 1883 que el objeto de estudio de la Geografía debía ser la "superficie terrestre", en una época con fuerte gravitación de la visión naturalista. Desde mediados del siglo XX, cuando la Geografía Humana comenzó a dimensionarse, los geógrafos enfatizaron en que su ciencia tenía que centrarse en el estudio del "espacio geográfico".

Este concepto es continente de otros, como región, paisaje, territorio o lugar. "Entendemos al espacio geográfico como la categoría más abstracta, resumen y expresión de la relación sociedad-naturaleza. Es una noción utilizada para referirse al escenario de la vida y del trabajo de las sociedades, y engloba al conjunto de los procesos de reproducción, en sentido amplio, de las distintas sociedades del globo." (Brunet et al, 1993. En: Gurevich, 2005)

Del término región, los geógrafos y economistas han hecho uso y abuso, situación que ocasionó una gran confusión plagada de imprecisiones conceptuales. La amplitud de las concepciones esgrimidas, hacen ver a la región, al mismo tiempo, como un tema o problema de estudio, una categoría de análisis o unidad de trabajo, un enfoque epistemológico y un método de investigación.

También la profusa literatura muestra una diversidad de tipos de regiones: naturales (por ejemplo: geológicas, climáticas, edafológicas, biogeográficas, ecológicas o eco-regiones), históricas, económicas, urbanizadas, etc., a las que se podría categorizar como regiones sistemáticas cuando están definidas por la homogeneidad de un elemento. Pero, siguiendo a Rey Balmaceda (1977), también se encuentran regiones administrativas, definidas por la decisión de una autoridad burocrática (organismo, empresa, entidad) como ocurre con la región-plan (estatal) y la regiónprograma (privada). No obstante, en esta disciplina, lo más frecuente es trabajar con regiones geográficas, las cuales pueden estar delimitadas por la conformidad de varios elementos (regiones formales) o por la cohesión funcional de un espacio (regiones funcionales o polarizadas).

Publicado en formato digital: Dr. Dante Edín Cuadra y otros. REGIÓN Y TERRITORIO. Aspectos conceptuales en torno a la organización del espacio y el rol de la ciencia geográfica. Revista Geográfica Digital. IGUNNE. Facultad de Humanidades. UNNE. Año 12. N N 24. Julio - Diciembre 2015. ISSN 1668-5180 Resistencia, Chaco. 
Otro aspecto que aumenta la complejidad del término está vinculado con las escalas posibles del tratamiento regional. Si bien se acepta que la región tiene un alcance subcontinental, se evidencia que presenta un amplio rango espacial, ya que puede abarcar un área o unidad terrestre que involucra a varios países o partes de ellos, una porción de un país (conformada por varias provincias) o, simplemente, áreas intra-provinciales. Esto significa que la extensión de una región geográfica puede variar sustancialmente según la escala escogida para identificar las unidades espaciales.

Una de las definiciones más conocidas ha sido enunciada por Daus (1982), quien consideraba que la región geográfica es“...un espacio continuo que, por diversas características provenientes del medio físico, biótico o de la actividad humana adquiere, en el tiempo, coherencia interna y unidad funcional."

Es importante tener en cuenta que la esencia de lo regional está dada por el rol que desarrolla la sociedad. En ese sentido, Labasse (1973) entendía que “...la personalidad de las regiones es más una obra del hombre que de la naturaleza” y que“...el hombre se expresa en el paisaje...”. Asimismo, Dickinson (1961) afirmaba que la región, como área intermedia entre las unidades de la administración local y el Estado, debe ser entendida “...como una asociación geográfica de las relaciones humanas en el espacio". También Benko (1999) ha dejado en claro que la región "...es un producto social construido progresivamente por las sociedades en sus espacios de vida", por lo tanto, es la acción humana la que le otorga organización e identidad.

Lajugie et al (1979) expresaba que "La región corresponde a un área geográfica que constituye una entidad que permite a la vez, la descripción de fenómenos naturales y humanos, el análisis de datos socio-económicos y la aplicación de una política."

Otros conceptos muestran la diversidad de apreciaciones, que se forjan en torno a la región: para Grigg “...la regionalización es un medio para alcanzar un fin, no un fin en sí mismo.” (Grigg, 1974. En Bruniard, 1995); para Bruniard (1995) "La región es una clase de espacio..." y, para Gottman (1977), una región geográfica “...puede siempre definirse por su red o tejido de encrucijadas.”

Si se pretende incorporar algo más de conflictividad al término, Pickenhayn (1994) sostiene que "Todos los que alguna vez intentaron dividir un país o continente en regiones, habrán descubierto por sí mismos que esta tarea es imposible: el resultado siempre es una convención discreta, producto de la acomodación subjetiva de un difícil rompecabezas..." Isard (1975), por su parte, ha afirmado que "...el concepto de región es engañoso..." y que ésta es una "...simple generalización del espíritu." Al decir de James (1952), "Cuando un geógrafo propone un sistema de divisiones regionales para ser utilizado en el estudio de un problema, de hecho está formulando una hipótesis."

También debe tomarse en cuenta que la connotación del término regional ha ido variando con el tiempo, tal como lo plantea Antón (1999), quien destaca que en el siglo XIX predominaba un criterio histórico-político en su consideración; luego, en tiempos de Vidal de la Blache (fines del siglo XIX y principios del XX) aún era relevante la caracterización física, posiblemente por la gravitación ejercida por la corriente positivista. Posteriormente, con la escuela norteamericana, tomó fuerza la concepción del contexto socio-económico para la ordenación del territorio. De hecho, la escuela francesa, en la segunda mitad del siglo XX, ya había desarrollado en su concepción historicista-posibilista del espacio, una clara preocupación por su ordenación. Hacia finales del siglo XX y en el tramo transitado del XXI la concepción de región se solapa, en ocasiones se sublima y, por momentos, 'anida en`o 'es anidada por'el concepto de territorio.

Benedetti (2009) expresa que "En el pensamiento geográfico, la categoría región adoptó tres sentidos fundamentales: división de un territorio, construcción política e identitaria e instrumento conceptual. A su vez, se pueden reconocer seis perspectivas conceptuales diferentes sobre la región: fisiográfica, humana, funcionalista, humanista, económico-política y político cultural."

\section{Planificación y desarrollo (regional / territorial)}

Puede asegurarse, sin vacilaciones, que "el planning tiene inevitablemente una base geográfica." (Freemann, 1958. En: Labasse, 1973), “...las estadísticas, empezando por las demográficas, son el

Publicado en formato digital: Dr. Dante Edín Cuadra y otros. REGIÓN Y TERRITORIO. Aspectos conceptuales en torno a la organización del espacio y el rol de la ciencia geográfica. Revista Geográfica Digital. IGUNNE. Facultad de Humanidades. UNNE. Año 12. N N 24. Julio - Diciembre 2015. ISSN 1668-5180 Resistencia, Chaco. 
primer paso para todo conocimiento regional." Pero, lo cuantitativo es una parte del análisis, el cual quedaría incompleto, desbalanceado y sesgado si no estuviese presente el componente cualitativo. "Lo cualitativo tiene, además, una virtud propia, la de invitarnos a considerar el factor sicológico, espiritual, cuya ignorancia o negligencia explica los mayores fracasos de las políticas planificadoras." (Labasse, 1973).

El estadio o fase en el que naturalmente debe desenvolverse el geógrafo es el de la planificación, pues allí tiene la posibilidad de volcar sus conocimientos técnico-disciplinares y de proponer cambios, elementos organizativos y funcionales en el espacio regional objeto de tratamiento. Las instancias siguientes, de intervención directa, son prerrogativas que recaen en el componente político-técnico de los organismos encargados de efectivizar lo planificado.

Cuando se habla de desarrollo, se hace alusión a la mejora de calidad de vida que experimenta la población de una región o de un territorio, gracias a las medidas y acciones llevadas adelante por el Estado (muchas veces asociado con sectores privados o constituyendo organizaciones mixtas) a lo largo de un período. Jordán y Sabatini (1988) enuncian un concepto extraído de un documento del Gobierno de El Salvador, que define al "desarrollo territorial" como un "Proceso de cambio progresivo que propicia la armonía entre el bienestar de la población, el uso del territorio, la conservación y protección de los recursos naturales y de las actividades productivas, a efecto de lograr el mejoramiento en la calidad de vida de la población, bajo un enfoque de sustentación."

Desde la visión de Precedo Ledo (2004), el desarrollo territorial es “...una estrategia de planificación para conseguir un desarrollo territorial equilibrado y sostenible basado en los recursos disponibles." Pero para ello, es necesario acompañar a las políticas económicas y territoriales de la dimensión sociocultural y sociopolítica, lo cual conduce a la formulación de un modelo de desarrollo sostenible que integre las políticas territoriales, económicas, sociales, culturales y medioambientales en un modelo único, basados en los principios de la coordinación, la subsidiariedad, la complementariedad y la cooperación..."

\section{Territorio, un concepto amplio y polisémico}

El concepto de territorio también ha experimentado cambios a través del tiempo. Tradicionalmente, en las ciencias humanas, estuvo asociado a la noción de espacio concreto perteneciente a un Estado, provincia o municipio, es decir, depositario de las dimensiones físicas, políticas, jurídicas, institucionales y demográficas. En la actualidad, un territorio es eso y mucho más. Se considera que es un producto social, más que eso, una "instancia de la totalidad social" (Blanco, 2009), es decir, que vuelve a incidir sobre la sociedad y la retroalimenta.

"Territorio es la categoría que contempla el espacio geográfico apropiado, puesto en valor y en el que se advierten las condiciones de un ejercicio efectivo del poder político. El territorio alude al espacio efectivamente usado, tasado o en reserva, resumen de las relaciones históricas entre la sociedad y la naturaleza." (Moraes, 1994 y Da Costa, 1993. En Gurevich, 2005) “...la noción de territorio es a la vez jurídica, social, cultural y también afectiva." "El territorio tiende a la proyección sobre un espacio dado de las estructuras específicas de un grupo humano, que incluyen el modo de división y de gestión del espacio, el ordenamiento de ese espacio." (Brunet et al, 1993. En Blanco, 2009).

Dice Fernández Caso (2009) que “...la organización del territorio tiene una dimensión histórica y política, que los procesos son protagonizados por diferentes sectores de la sociedad, que las distintas sociedades nacionales se relacionan entre sí y que esta relación es distinta en cada momento histórico, cada vez más intensa en el momento actual, y que de todo ello resultan configuraciones diversas, pero no aleatorias."

Otros aportes vertidos por Blanco (2009) resultan muy interesantes y esclarecedores, en primer lugar que "...el concepto de territorio lleva implícitas las nociones de apropiación, ejercicio del dominio y control de una porción de la superficie terrestre, pero también contiene las ideas de pertenencia y de proyectos que una sociedad desarrolla en un espacio dado". En su análisis este autor sostiene que

Publicado en formato digital: Dr. Dante Edín Cuadra y otros. REGIÓN Y TERRITORIO. Aspectos conceptuales en torno a la organización del espacio y el rol de la ciencia geográfica. Revista Geográfica Digital. IGUNNE. Facultad de Humanidades. UNNE. Año 12. N N 24. Julio - Diciembre 2015. ISSN 1668-5180 Resistencia, Chaco. 
"La apropiación y transformación de un espacio por parte de una sociedad implica entonces la construcción de un territorio..." y cita a Raffestin (1993), quien afirma que "el territorio se forma a partir del espacio." Luego, hace referencia al "sistema de normas" como "un conjunto de regulaciones que organizan el funcionamiento de un territorio" y a la relevancia que tienen las "redes" como "elementos constitutivos del territorio" que "traen inevitablemente una perspectiva relacional, ya que remiten de manera inequívoca a relaciones con otros territorios" y posibilitan la "articulación de escalas", resaltando la aseveración de Pumain y Saint-Julien (2004), quienes postulan que "No existe territorio sin red."

Los autores Gómez Orea, Gómez Villarino, A. y Gómez Villarino, M. T. (2014) recurren al concepto de 'sistema territorial y sostienen que es una construcción social que se relaciona con el desarrollo de una sociedad. Esa construcción es permanente, sobre la base de las actividades de la población sobre el medio físico y las interrelaciones que se dan entre ellos y, además, se halla normada. Esta concepción es coincidente con expresiones vertidas en párrafos anteriores. Dichos autores afirman que, en el 'sistema territorial', es posible detectar tres elementos que constituyen la sustancia del sistema y se agrega un cuarto relacionado con lo temporal. Los elementos sustanciales son:

- Estructura: son los componentes del sistema, abarcan tanto el medio físico, pasando por la población, actividades humanas, asentamientos, relaciones, instituciones, agentes y marco legal.

- Funcionamiento: se expresan a través de los flujos donde se destaca la infraestructura de transportes y de las telecomunicaciones. En ese elemento se presentan las relaciones, tanto hacia el exterior como hacia el interior.

- Imagen externa: representa la base del paisaje y la percepción polisensorial y subjetiva.

- Tiempo: señala la forma en que evoluciona el sistema y los mecanismos de control para adaptarse, porque el objetivo es permanecer.

Es interesante destacar la perspectiva de Haesbaert (2007), al explicitar que "El gran dilema de este inicio de milenio...(es) el de la multiterritorialización,...experimentar diferentes territorios al mismo tiempo, reconstruyendo constantemente el nuestro". "El territorio envuelve siempre, al mismo tiempo...una dimensión simbólica, cultural, a través de una identidad territorial atribuida por los grupos sociales, como forma de 'control simbólico`sobre el espacio donde viven..."

\section{Gestión territorial}

Incluye la totalidad de las acciones políticas, administrativas, económico-financieras y técnicas desde la instancia misma de tomar la decisión de ordenar un territorio, el proceso durante el cual éste se lleva a cabo, prolongándose después de su ejecución hasta cuando se considere necesario realizar monitoreos, controles y ajustes.

Contempla el diseño de los circuitos administrativos que deben seguirse, la generación de los vínculos interinstitucionales nacionales, internacionales, regionales y locales, las relaciones con entes privados, búsquedas de financiamiento y cooperación, negociaciones políticas para el logro de acuerdos y consensos (para la aprobación de nuevas normativas, por ejemplo) con legisladores, gobiernos locales y referentes sociales, promoción de la participación ciudadana, entre otras.

En definitiva, se trata del "conjunto de procesos que se deben implementar... para hacer realidad la construcción del modelo territorial de desarrollo planteado,... mediante la integración de recursos humanos, financieros, organizacionales, políticos y naturales, buscando satisfacer las necesidades individuales y colectivas de los diferentes sectores de la población..." Ello requiere la conformación de: a) una "plataforma institucional" (que"comprende el conjunto de estrategias y acciones a desarrollar, con el fin de adecuar la estructura organizativa...y elevar su capacidad de gestión...para asumir un papel de dinamizador, promotor y facilitador; para asegurar que los propósitos del plan de ordenamiento se cumplan con el concurso y la vinculación de los diferentes actores, valga decir:

Publicado en formato digital: Dr. Dante Edín Cuadra y otros. REGIÓN Y TERRITORIO. Aspectos conceptuales en torno a la organización del espacio y el rol de la ciencia geográfica. Revista Geográfica Digital. IGUNNE. Facultad de Humanidades. UNNE. Año 12. N N 24. Julio - Diciembre 2015. ISSN 1668-5180 Resistencia, Chaco. 
entidades públicas..., el sector privado, el comunitario y la academia"; b) una "plataforma físicoespacial" ("conformada por las estrategias, políticas y acciones y/o proyectos relacionados con las intervenciones que se deben hacer sobre el suelo y los diferentes sistemas estructurantes (servicios públicos, vías y transporte, espacio público, vivienda, etc.), para alcanzar la visión de futuro; todos ellos recogidos en el modelo territorial del componente general y los modelos de los componentes urbano y rural..."(Municipio de Páramo-Santander, 2002. "Gestión Esquema Ordenamiento Territorial E.O.T.”. Colombia).

\section{Planificación, planeamiento o planeación territorial}

Los términos planeamiento y planeación tienen igual significación, sólo que el primero es de uso más generalizado, en tanto que el segundo se utiliza sólo en algunos países, como México y Guatemala. La connotación de estos términos alude al trazado de planes y proyectos en el sentido de ideas, políticas y diseños organizacionales, trazos generales de obras, estrategias y mecanismos formales y legales que deben respetarse en los ámbitos administrativos y empresariales. El término planificación suele utilizarse con otra significación, más vinculada a planes con mayores exigencias técnicas, alto grado de detalle (exhaustivo) y metódico que obligatoriamente exige la incorporación de un cronograma de acciones que prevén desarrollarse.

No obstante, en la mayoría de los países (y sus jurisdicciones menores como las provincias, municipios y entes regionales) se usan indistintamente estas denominaciones para identificar a los organismos encargados de resolver las problemáticas territoriales. Así encontramos ministerios, secretarías o direcciones de Planeamiento (Planeación) o de Planificación que desarrollan funciones similares.

En este trabajo se ha preferido utilizar el término planificación, no sólo por ser el más difundido, sino porque -en Argentina- ha cobrado relevancia en las últimas décadas, al tiempo que el término planeamiento ha quedado un tanto relegado y es depositario de una connotación, en cierto modo, estanca y de aplicación más administrativa que técnica.

La planificación representa una herramienta que incluye componentes técnicos, sociales y políticos, cuya misión es diseñar el uso racional (sustentable) del suelo, contemplando que los grupos humanos alcancen un pleno desarrollo. Es una parte relevante dentro del ordenamiento territorial, donde la política debe dar los espacios suficientes a la técnica (trabajos interdisciplinarios de diferentes profesionales). La planificación física puede ser territorial (a una escala regional o local) y sectorial (específicamente de un sector o función, como las áreas agrícolas, los equipamientos o los espacios recreativos de un centro urbano). En el primer caso, están consideradas la planificación estratégica y la planificación urbano-ambiental.

La planificación en la actualidad, a diferencia de la tradicional, desde la perspectiva de Jordán y Sabatini (1988), se caracteriza por una mayor operatividad, flexibilidad y eficiencia, es más innovadora y, por tanto, más dinámica, incluye componentes estratégicos, nuevos y renovados instrumentos, programas, proyectos, normas, modos de gestión y formas de participación que la hacen portadora de mayor legitimidad. Asimismo, se plantea nuevas prioridades que incluyen la adopción de nuevos principios y objetivos.

En cuanto a la planificación, Gómez Orea junto a otros autores (2014), sostienen que todo sistema territorial evoluciona y puede llevarse a cabo de dos modos:

- Espontánea o Tendencial: aquí se refleja la acción conjunta de las fuerzas naturales y sociales. Los problemas se resuelven en beneficio del sector privado o del considerado más fuerte y a corto plazo. Hay una planificación, porque alguien piensa estas acciones. Ese territorio presenta características y dan como resultado un 'territorio indeseado'.

- Planificada: consecuencia de un proceso racional de toma de decisiones, tiene por resultado un 'territorio satisfactorio', que constituye la esencia de la ordenación.

Publicado en formato digital: Dr. Dante Edín Cuadra y otros. REGIÓN Y TERRITORIO. Aspectos conceptuales en torno a la organización del espacio y el rol de la ciencia geográfica. Revista Geográfica Digital. IGUNNE. Facultad de Humanidades. UNNE. Año 12. N N 24. Julio - Diciembre 2015. ISSN 1668-5180 Resistencia, Chaco. 
Revista Geográfica Digital. IGUNNE. Facultad de Humanidades. UNNE. Año 12. № 24.

Julio - Diciembre 2015. ISSN 1668-5180 Resistencia, Chaco

\section{Ordenamiento u ordenación territorial}

El geógrafo francés Jean Labasse se encargó de clarificar, en la década de 1970, que el concepto de "ordenación" (hoy tan utilizado en la literatura geográfica como si se tratara de un descubrimiento reciente), representa "...una contracción de uso más cómodo y habitual" del término "organización espacial", siempre asociado al tratamiento de los problemas de repartición y mejor utilización del terreno, que apunta -mediante la implementación de una política localizadora- al logro de mejores condiciones, niveles y calidad de vida del grupo humano que lo habita.

Como bien explica Parrado Delgado (2001), la palabra "ordenación territorial" (equivalente al término "ordenamiento territorial", de uso más corriente en Argentina) tuvo su origen en Francia a fines de la década de 1940, en el propio Ministerio de la Reconstrucción y del Urbanismo, ante el escenario de desorden espacial producto de las acciones bélicas desplegadas durante la segunda guerra mundial. No obstante, su implementación efectiva (sanción de leyes y aprobación de planes concretos de intervención) se produjo recién en 1967 en aquél país.

El mencionado autor, resalta la importancia que tuvo la Carta Europea de Ordenación del Territorio, rubricada por la Conferencia Europea de Ministros Responsables de la Ordenación del Territorio (CEMAT) en 1988, documento en el cual se concibe a la ordenación territorial como la "proyección espacial de las políticas sociales, culturales, ambientales y económicas de una sociedad".

En Europa la ordenación territorial es respaldada por el orden supranacional, luego reglamentada en los niveles intermedios, para su posterior aplicación en los niveles inferiores, de manera específica bajo la forma de planeamiento urbanístico. Su práctica demanda tres condiciones:

- Voluntad política: cuyo objetivo es el control del uso del suelo de acuerdo con la función social de la propiedad.

- $\quad$ Cobertura legal de los planes: normativa vinculante.

- $\quad$ Capacidad institucional: formular planes y gestionarlos.

Es muy importante saber, antes de seguir avanzando, que la ordenación territorial es -al mismo tiempo- una disciplina científica, una técnica administrativa y una política, entendida como actuación interdisciplinaria y global, cuyo objetivo es un desarrollo equilibrado de regiones y la organización física del espacio. (Gómez Orea, 1992. En Parrado Delgado, 2001)

El eminente geógrafo español Casas Torres, hace más de cuatro décadas, sostenía que “...contribuir a la ordenación del espacio es la principal tarea de la geografía aplicada" y que las regiones son la "pieza clave de la ordenación del espacio y la planificación del desarrollo". (Casas Torres, 1973. En: Labasse, 1973). En el mismo texto se cita a Prothin (1955), quien afirmaba que "La ordenación exige un conocimiento profundo de geografía económica, como de geografía humana".

Casas Torres, a comienzos de la década de 1970, afirmaba que el rol del geógrafo es primordial en los estudios previos de un espacio, sus habitantes y su trabajo en toda su delicada complejidad, como punto de partida de la ordenación territorial y, asimismo, el seguimiento de lo que va sucediendo como resultado de esa ordenación, lo que implica desarrollar tareas en equipos en los cuales los geógrafos forman parte junto a otros profesionales.

El mencionado autor enfatizaba en la necesidad de un sentido de lo espacial, de la correlación de todos los hechos que se registran sobre un mismo territorio, que complete las visiones parciales (analíticas, sectoriales) de los científicos especialistas. Resaltaba que esa visión sintética y locacional es propia de los profesionales de la Geografía, pero al mismo tiempo reconocía (y se autoinculpaba) que los geógrafos habían dejado un amplio flanco sin cubrir que fue ocupado por colegas sin sentido de lo espacial, ni de lo específicamente geográfico, atribuyendo esa falencia a varias causas: demasiado cientificismo en desmedro de las tareas de divulgación, pudor y pereza en escribir para el gran público y, por último, exceso de trabajo docente (de horas de clase y administrativo). Lo cierto es que han pasado más de cuarenta años de estas observaciones o llamados de atención para los geógrafos y, en algunos países como el nuestro, poco han sido los cambios ocurridos.

Publicado en formato digital: Dr. Dante Edín Cuadra y otros. REGIÓN Y TERRITORIO. Aspectos conceptuales en torno a la organización del espacio y el rol de la ciencia geográfica. Revista Geográfica Digital. IGUNNE. Facultad de Humanidades. UNNE. Año 12. № 24. Julio - Diciembre 2015. ISSN 1668-5180 Resistencia, Chaco. 
El ordenamiento territorial ha sido considerado como la expresión espacial de las políticas públicas. Jordán y Sabatini (1988) lo definen como "Un conjunto de acciones concertadas para orientar la transformación, ocupación y utilización de los espacios geográficos buscando su desarrollo socioeconómico, teniendo en cuenta las necesidades e intereses de la población, las potencialidades del territorio considerado y la armonía con el medio ambiente". En definitiva, se trata de una función pública y, más específicamente, de un "...instrumento de acción pública...", que se compone de varias etapas: análisis territorial (recolección y tratamiento de la información), diagnóstico (examen de la situación actual, determinación de los objetivos que se desean alcanzar, estudios de pre-factibilidad y factibilidad), planificación (planteamiento de los problemas que se quieren resolver, elaboración de las propuestas o situación deseada), ejecución (implementación en el terreno) y seguimiento (evaluación periódica y realización de ajustes).

Dicen Jordán y Sabatini (1988) que "El plan de Ordenamiento Territorial en la actualidad, de carácter estratégico, ya no debe ser concebido tan solo como una norma sino...como un proceso vinculado a un programa de acción territorial. Se trata de un nuevo instrumento de visión perspectiva, de contenidos específicos y de acciones concretas que se desarrollan a través del tiempo."

El ordenamiento territorial es una función básica de los poderes públicos para resolver conflictos de interés común, no solo los actuales sino que también tiende a prevenir los potenciales. Esta tarea se ampara en el concepto de desarrollo sostenible, además de la participación de agentes socioeconómicos.

El ordenamiento territorial, enlazando con lo dicho anteriormente y de acuerdo con Gómez Orea, Gómez Villarino, A. y Gómez Villarino, M. T. (2014), implica el cumplimiento de tres facetas complementarias:

- Diagnostico territorial: estado de situación.

- $\quad$ Planificación territorial: sistema territorial a que se aspira.

- $\quad$ Gestión territorial: acciones para alcanzar el sistema territorial deseado.

Desde la perspectiva de estos autores, el ordenamiento territorial opera a través de un conjunto coherente de planes o instrumentos, de carácter integral y sectorial, previstos tanto en una ley específica como en leyes sectoriales. Esos planes pueden organizarse en:

- $\quad$ Cascada: los planes de nivel superior se toman como referencia para los de nivel inferior. Se refiere a los ámbitos geográficos.

- $\quad$ Contracorriente: hay una relación desde el superior al inferior y a la inversa.

- $\quad$ Coherencia: a cada nivel le corresponde determinadas competencias.

- Integración vertical: cada una se integra hacia arriba y hacia abajo.

- $\quad$ Subsidiariedad: el ciudadano no está ajeno a las decisiones.

- Incorporación de: transparencia, participación de los ciudadanos y concertación de los agentes socioeconómicos.

La primera tarea que debe realizar un profesional, antes de realizar un plan de ordenamiento territorial, es revisar y estudiar la legislación referida a la ordenación en su país.

Un plan de ordenamiento territorial, a la vez, tiene vinculación con otros instrumentos:

- Otros planes: integrales por áreas homogéneas, funcionales o estratégicas y, por otra parte, los sectoriales (definidos por problemas, extensión o potencialidad).

- $\quad$ Normativa general y particular: establece los usos y aprovechamientos del suelo.

- Programación: intervenciones organizadas en niveles de concreción, como programas y proyectos.

- Gestión de organismos responsables, sistema de gestión e indicadores para el seguimiento.

- $\quad$ Financieros: proveen los recursos económicos para su implementación.

- $\quad$ Evaluación "ex post": con el objetivo de medir el comportamiento del plan.

Publicado en formato digital: Dr. Dante Edín Cuadra y otros. REGIÓN Y TERRITORIO. Aspectos conceptuales en torno a la organización del espacio y el rol de la ciencia geográfica. Revista Geográfica Digital. IGUNNE. Facultad de Humanidades. UNNE. Año 12. № 24. Julio - Diciembre 2015. ISSN 1668-5180 Resistencia, Chaco. 
- $\quad$ Reformulación: para corregir posibles inconvenientes del plan y, paralelamente, aplicar el principio de planificación continua.

- Gestión ambiental: cuyo objetivo es lograr una elevada calidad ambiental. (Gómez Orea et al, 2014)

\section{Consideraciones finales}

El espacio geográfico constituye una categoría inclusiva de otros conceptos usuales en Geografía y otras ciencias, tales como región y territorio (tratados en este artículo), paisaje (como reflejo, fisonomía o aspectos perceptibles de un espacio) y lugar (vinculado con el espacio vivencial, simbólico, emotivo e identitario). Cada uno de ellos cobra mayor fuerza según la perspectiva teórica o epistemológica desde la que operamos dentro de nuestras disciplinas. Lo que queda claro es que estos conceptos no se agotan desde una mirada disciplinar y, necesariamente, requieren una visión integradora, algunas veces interdisciplinar y, otras, transdisciplinar.

Tanto la región como el territorio, más allá de los aspectos difusos que podemos encontrar a nivel teórico-conceptual, debido a las variadas vertientes que participan en su estudio y análisis, sumadas a la propia dinámica que experimentan las definiciones, alcances y enfoques de los conceptos, representan un campo pertinente a la Geografía, imposible de eludir en la época de cambios espaciales que transitamos. Organizar la región, contribuir a su desarrollo, planificar y ordenar los territorios son cuestiones relevantes socialmente, desafíos reales en los que la ciencia geográfica debe estar siempre representada, trabajando codo a codo con ingenieros, arquitectos, urbanistas, biólogos, cientistas políticos, economistas y sociólogos, entre otros.

Es notable cómo el tema de la organización espacial ha vuelto a cobrar vigencia, quizás como nunca después de la segunda guerra mundial y, la Geografía como ciencia encargada de estudiar el espacio geográfico, no puede desentenderse en esta urgente e ineludible búsqueda del orden espacial, tanto regional como territorial. Tamaña tarea nos impone el desafío de operar en sociedades y territorios que tienen como "principales atributos" la "complejidad", la "fragmentación", la "desigualdad", la "diferencia", la "desterritorialización-reterritorialización" y "...hacer frente a los riesgos deshumanizantes, a la velocidad de los cambios y a la injusticia en sus variadas formas de exclusión y desamparo." (Gurevich, 2005)

Como ciencia humanística y social, la Geografía se halla en el momento justo para dar el salto de calidad esperado, enfocándose y ofreciendo respuestas apropiadas a las problemáticas territoriales tan acuciantes de nuestro tiempo. 


\section{Bibliografía}

Antón, J. (1999). Regiones económicas argentinas. Ciudad Argentina. Editorial de Ciencia y Cultura. Madrid, España.

Benedetti, A. (2009). Los usos de la categoría región en el pensamiento geográfico argentino. Scripta Nova. Revista electrónica de Geografía y Ciencias Sociales. Nueva serie deGeo Crítica. Cuadernos Críticos de Geografía Humana. Vol XIII, № 286. Universidad de Barcelona. Barcelona, España.

Benko, G. (1999). La Ciencia Regional. Colección Sociedad y Territorio. Ed. de la Universidad Nacional del Sur. Serie Extensión. Bahía Blanca, Argentina.

Berciano Villalibres, M. (1998). Debate en torno a la Posmodernidad. Ed. Síntesis. Hermeneia. Madrid, España.

Blanco, J. (2009). Espacio y territorio: elementos teórico-conceptuales implicados en el análisis geográfico. En: Fernández Caso, M.V. y Gurevich, R. (Coordinadoras). "Geografía. Nuevos temas, nuevas preguntas. Un temario para su enseñanza". Ed. Biblos. Colección Claves para la Formación Docente. Buenos Aires, Argentina.

Brunet, R. et al (1993). Les mots de la Géographie. En: Blanco, J. (2009). "Espacio y territorio: elementos teórico-conceptuales implicados en el análisis geográfico". En: Fernández Caso, M. V. y Gurevich, R. (Coordinadoras). "Geografía. Nuevos temas, nuevas preguntas. Un temario para su enseñanza". Ed. Biblos. Colección Claves para la Formación Docente. Buenos Aires, Argentina.

Brunet, R. et al (1993). Les mots de la Géographie. En: Gurevich, R. (2005). "Sociedades y territorios en tiempos contemporáneos. Una introducción a la enseñanza de la geografía". Fondo de Cultura Económica S. A. Buenos Aires, Argentina.

Bruniard, E. (1995). Las regiones homogéneas en Geografía Humana (Guía de trabajos prácticos). Nordeste 2da. Época. Serie: Docencia, № 1 Geografía. Facultad de Humanidades, Universidad Nacional del Nordeste. Resistencia, Argentina.

Daus, F. (1982). Fundamentos para una división regional de la Argentina. En: Aportes al Pensamiento Geográfico. № 1. Sociedad Argentina de Estudios Geográficos (1990). Buenos Aires, Argentina.

Dickinson, R. (1961). Ciudad, región y regionalismo. Ed. Omega S.A. Barcelona, España.

Fernández Caso, M. V. (2009). Discursos y prácticas en la construcción de un temario en geografía. En: Fernández Caso, M. V. y Gurevich, R. (Coordinadoras). "Geografía. Nuevos temas, nuevas preguntas. Un temario para su enseñanza". Ed. Biblos. Colección Claves para la Formación Docente. Buenos Aires, Argentina.

Foucault, M. (2006). Seguridad, territorio, población. Sección de Obras de Sociología. Fondo de Cultura Económica. México D.F.

Freemann, T. (1958). Geography and planning. En: Labasse, J. (1973). "La organización del espacio. Elementos de Geografía Aplicada". Instituto de Estudios de Administración Local. Malvar S.A., Madrid, España.

Gómez Órea, D. (1992). Documentos para la asignatura en Recursos Naturales y Ordenación del Territorio. En: Parrado Delgado, C. (2001). "Metodología para la ordenación del territorio bajo el prisma de sostenibilidad (estudio de su aplicación en la ciudad de Bogotá D.C.)". Tesis Doctoral. Departament d’ Enginyeria Minera i Recursos Naturals. Universidad Politècnica de Catalunya. Barcelona, España.

Gómez Orea, D., Gómez Villarino, A. y Gómez Villarino, M. T. (2014). Marco legal e institucional de la ordenación territorial: instrumentos generales y específicos. I Curso Internacional de Postgrado de Ordenamiento Territorial. Modalidad virtual. Fundabaires. Buenos Aires, Argentina.

Publicado en formato digital: Dr. Dante Edín Cuadra y otros. REGIÓN Y TERRITORIO. Aspectos conceptuales en torno a la organización del espacio y el rol de la ciencia geográfica. Revista Geográfica Digital. IGUNNE. Facultad de Humanidades. UNNE. Año 12. N N 24. Julio - Diciembre 2015. ISSN 1668-5180 Resistencia, Chaco. 
Gottman, J. (1977). El método de análisis en Geografía Humana. En: Teoría de la Geografía. Primera Parte. Sociedad Argentina de Estudios Geográficos. Serie Especial № 3. Buenos Aires, Argentina.

Grigg, D. (1974). Regioes, Modelos e Classes. En: Bruniard, E. (1995). "Las regiones homogéneas en Geografía Humana (Guía de trabajos prácticos). Nordeste 2da. Época. Serie: Docencia, $N^{0} 1$ Geografía. Facultad de Humanidades, Universidad Nacional del Nordeste. Resistencia, Argentina.

Gurevich, R. (2005). Sociedades y territorios en tiempos contemporáneos. Una introducción a la enseñanza de la geografía. Fondo de Cultura Económica S. A. Buenos Aires, Argentina.

Haesbaert, R. (2007). O mito da desterritorialização: do "fim dos territórios" á multiterritorialidade. $3^{\circ}$ ed. Bertrand. Rio de Janeiro, Brasil.

Isard, W. (1975). Introduction to Regional Science. Prentice-Hall. Englewood Cliffs, New Jersey, USA.

James, P. (1952). Hacia una más profunda comprensión del concepto regional. En: Randle, P., Editor. (1977). Teoría de la Geografía. GAEA. Buenos Aires, Argentina.

Jordán, R. y Sabatini, F. (1988). Economía política de los desastres naturales: prevención y capacitación. Revista EURE. Vol. 14, № 43. Santiago, Chile.

Labasse, J. (1973). La organización del espacio. Elementos de Geografía Aplicada. Instituto de Estudios de Administración Local. Malvar S. A., Madrid, España.

Lajugie, J. et al (1979). Espace régional et aménagement du territoire. Dalloz. Paris, Francia.

Montes Lira, P. (2001). El ordenamiento territorial como opción de políticas urbanas y regionales en América Latina y el Caribe. Serie Medio Ambiente y Desarrollo. CEPAL-ECLAC. Naciones Unidas. División de Medio Ambiente y Asentamientos Humanos. Santiago, Chile.

Moraes, A. (1994). Meio Ambiente e Ciências Humanas; Da Costa, W. (1994). Geografia Crítica. A valorização do spaço. En: Gurevich, R. (2005). "Sociedades y territorios en tiempos contemporáneos. Una introducción a la enseñanza de la geografía". Fondo de Cultura Económica S. A. Buenos Aires, Argentina.

Municipio de Páramo-Santander (2002). Gestión Esquema Ordenamiento Territorial E.O.T. Colombia. $\quad$ En: http://paramo-santander.gov.co/apc-aafiles/65323236313364303735613264386434/1.GESTION_TERRITORIAL.pdf

Nogué, J. y Rufí, V. (2001). Geopolítica, identidad y globalización. En: Precedo Ledo, A. (2004). "Nuevas realidades territoriales para el siglo XXI. Desarrollo local, identidad territorial y ciudad difusa". Editorial Síntesis. Madrid, España.

Parrado Delgado, C. (2001). Metodología para la ordenación del territorio bajo el prisma de sostenibilidad (estudio de su aplicación en la ciudad de Bogotá D.C.). Tesis Doctoral. Departament d’Enginyeria Minera i Recursos Naturals. Universidad Politècnica de Catalunya. Barcelona, España.

Precedo Ledo, A. (2004). Nuevas realidades territoriales para el siglo XXI. Desarrollo local, identidad territorial y ciudad difusa. Editorial Síntesis. Madrid, España.

Prothin, A. (1955). En: Labasse, J. (1973). La organización del espacio. Elementos de Geografía Aplicada. Instituto de Estudios de Administración Local. Malvar S.A., Madrid, España.

Pumain, D. y Saint-Julien, T. (2004). L'Analyse spatiale. En: Blanco, J. (2009). "Espacio y territorio: elementos teórico-conceptuales implicados en el análisis geográfico". En: Fernández Caso, M. V. y Gurevich, R. (Coordinadoras). "Geografía. Nuevos temas, nuevas preguntas. Un temario para su enseñanza”. Ed. Biblos. Colección Claves para la Formación Docente. Buenos Aires, Argentina.

Publicado en formato digital: Dr. Dante Edín Cuadra y otros. REGIÓN Y TERRITORIO. Aspectos conceptuales en torno a la organización del espacio y el rol de la ciencia geográfica. Revista Geográfica Digital. IGUNNE. Facultad de Humanidades. UNNE. Año 12. N N 24. Julio - Diciembre 2015. ISSN 1668-5180 Resistencia, Chaco. 
Raffestin, C. (1993). Por uma Geografia do Poder. En: Blanco, J. (2009). "Espacio y territorio: elementos teórico-conceptuales implicados en el análisis geográfico". En: Fernández Caso, M. V. y Gurevich, R. (Coordinadoras). "Geografía. Nuevos temas, nuevas preguntas. Un temario para su enseñanza”. Ed. Biblos. Colección Claves para la Formación Docente. Buenos Aires, Argentina.

Rey Balmaceda, R. (1977). Geografía Regional. Teoría y aplicación. Ed. Estrada. Buenos Aires, Argentina. 